\title{
Correction to: The Role of Adsorbed and Subsurface Carbon Species for the Selective Alkyne Hydrogenation Over a Pd-Black Catalyst: An Operando Study of Bulk and Surface
}

\author{
J. J. Velasco-Vélez ${ }^{1,2} \cdot$ D. Teschner ${ }^{1,2} \cdot$ F. Girgsdies ${ }^{2} \cdot$ M. Hävecker ${ }^{1,2} \cdot$ V. Streibel ${ }^{2} \cdot$ M. G. Willinger ${ }^{2} \cdot$ J. Cao $^{2}$.

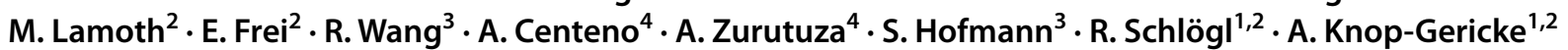

Published online: 20 November 2018

(c) The Author(s) 2018

\section{Correction to: Topics in Catalysis https://doi.org/10.1007/s11244-018-1071-6}

The original version of this article unfortunately contained an error. The authors would like to correct the error with this erratum. In Fig. 5 the processes $\mathrm{C}$ and $\mathrm{B}$ were incorrectly labelled. The corrected Fig. 5 with the right labelling is included in this erratum.

In addition the equations describing the chemical reactions were not included in the discussion in original text. The discussion including the equations is included in this erratum:

\section{Discussion}

According to the above described experiments, the existence of $\mathrm{PdC}_{\mathrm{x}}$ species, which are formed by alkyne decomposition and carbon diffusion into the Pd lattice [42], appears to be a key factor in the selective hydrogenation of alkynes into alkenes. Thus, $\mathrm{PdC}_{\mathrm{x}}$ species strongly disturbs the equilibrium between hydrogen dissolved in the bulk and adsorbed

The original article can be found online at https://doi.org/10.1007/ s11244-018-1071-6.

J. J. Velasco-Vélez

velasco@fhi-berlin.mpg.de

1 Department of Heterogeneous Reactions, Max Planck Institute for Chemical Energy Conversion, 45470 Mülheim an der Ruhr, Germany

2 Department of Inorganic Chemistry, Fritz-Haber-Institut der Max-Planck-Gesellschaft, 14195 Berlin, Germany

3 Department of Engineering, University of Cambridge, Cambridge CB3 OFA, UK

4 Graphenea, 20018 San Sebastian, Spain on the surface. The bulk hydrogen is much more reactive than the surface hydrogen, and can hydrogenate the adsorbed species upon their appearance on the surface, thus contributing to the unselective hydrogenation [44]. The incorporation of carbon into the upper Pd layers strongly influences the diffusion/transport of $\mathrm{H}$ from the bulk to the uppermost $\mathrm{Pd}$ layer, thereby hindering the participation of more energetic subsurface hydrogen in the catalytic process. On the other hand, increasing the hydrogen partial pressure promotes the unselective total hydrogenation of propyne to propane, which is accompanied by a decrease in the amount of surface $\mathrm{PdC}_{\mathrm{x}}$ and an enhancement of the surface $\mathrm{PdH}_{\mathrm{x}}$ species $[45,46]$. Furthermore, the role of $\mathrm{PdC}_{\mathrm{x}}$ was also deduced from operando EXAFS experiments [47]. In addition, these experiments proved the existence of different chemisorbed hydrogenated species depending on the dominant surface state $\left(\mathrm{PdC}_{\mathrm{x}}\right.$ or $\left.\mathrm{PdC}_{\mathrm{x}} \mathrm{H}_{\mathrm{y}}\right)$. That is, the chemisorbed hydrocarbon species are more dehydrogenated over $\mathrm{PdC}_{\mathrm{x}}$ and more hydrogen rich over $\mathrm{PdH}_{\mathrm{x}}$. Taking into account the above described processes, the general scheme of the hydrogenation of hydrocarbons with multiple unsaturations is linked to the so-called "rake mechanism" because the adsorption and desorption steps and equilibrium constant are like the "teeth" of a "comb" $[48,49]$. This process can be accurately described taking into account the chemistry of the population of palladium surface sites and the rate constant $(\mathrm{k})$ for each catalytic process [50]. Thus, two scenarios are considered: the partial hydrogenation on $\alpha-\beta \mathrm{PdC}_{\mathrm{x}}$ and the full hydrogenation on $\beta-\mathrm{PdC}_{\mathrm{x}} \mathrm{H}_{\mathrm{y}}$ catalysts, as shown in Fig. 5 . These processes are governed by carbon and/or hydrogen subsurface atoms, as the operando experiments showed. Hence, the processes $\mathrm{A}$ and a are related to the adsorption and desorption of $\mathrm{C}_{\mathrm{n}} \mathrm{H}_{2 \mathrm{n}-2}$ on the Pd surface yielding the formation of $\mathrm{PdC}_{\mathrm{x}}$ as main product of the reaction. Moreover, in presence of $\mathrm{C}_{n} \mathrm{H}_{n-2}$ and $\mathrm{H}_{2}$, two reactions are expected, which are described by the constant processes $\mathrm{B}$ 
Fig. 5 Proposed extended "rake" reaction mechanism accounting the formation of surface/subsurface $\mathrm{PdH}_{\mathrm{x}} / \mathrm{PdC}_{\mathrm{x}}$ and bulk $\beta$-PdHx species as well as chemisorbed $\mathrm{CnH}_{2 \mathrm{n}-2} / \mathrm{CnH}_{2 \mathrm{n}}$ intermediates

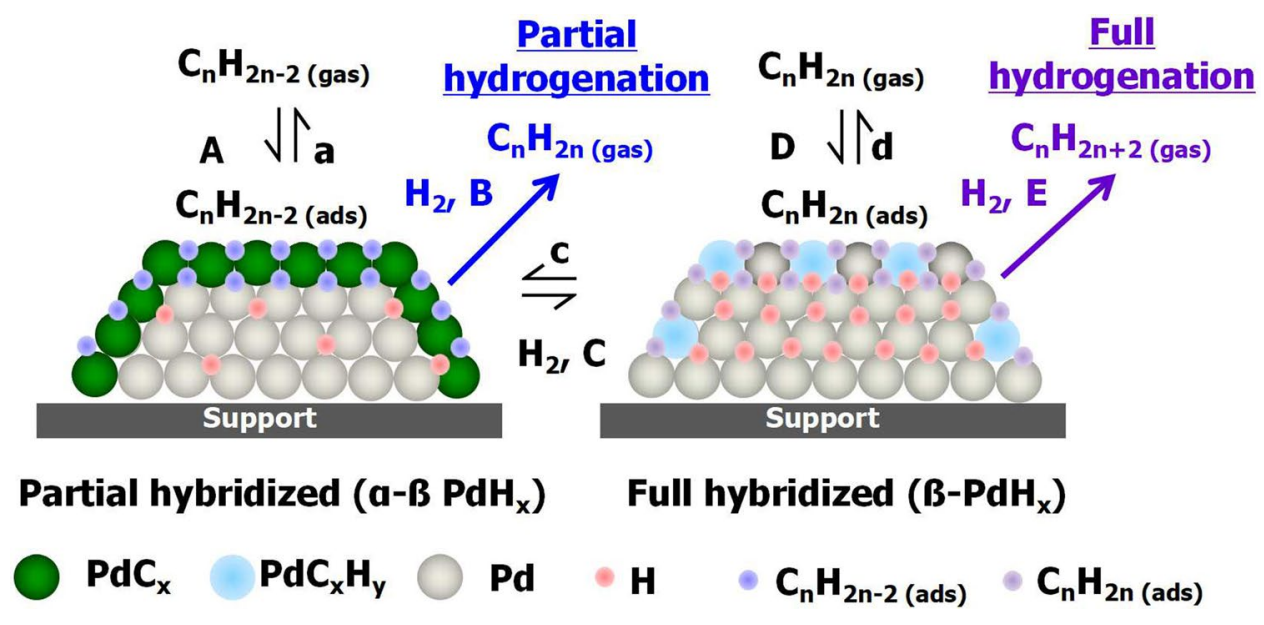

and $\mathrm{C}$, given that in the case of low $\mathrm{H}_{2}$ concentration $\mathrm{B}$ is the dominant rate constant. Hence low partial pressures of hydrogen yields:

$$
P d C_{x}+C_{n} H_{2 n-2(\text { Chemisorb. })}+H_{2} \underset{B}{\rightarrow} P d C_{x}+C_{n} H_{2 n(\text { gas })}
$$

This reaction is selectively due to the formation of $\mathrm{PdC}_{\mathrm{x}}$ by the incorporated carbon in the upper layers preventing the effective diffusion of rather reactive bulk/subsurface hydrogen. As a consequence, the upper carbonaceous species lead to the formation of alkenes avoiding the full hydrogenation on the Pd catalyst and thus hindering the $\mathrm{C}$ unselective mechanism. On the other hand, if the partial concentration of $\mathrm{H}_{2}$ is increased then $\mathrm{C}$ is the dominant prompting the hydrogenation of $\mathrm{PdC}_{\mathrm{x}}$ as:

$$
P d C_{x}+C_{n} H_{2 n-2(\text { Chemisorb. })}+H_{2} \underset{C}{\rightarrow} P d C_{x} H_{y}+C_{n} H_{2 n-2(\text { gas })}
$$

Once the $\mathrm{PdC}_{\mathrm{x}}$ surface concentration is lowered and the $\mathrm{PdH}_{\mathrm{x}}$ becomes dominant the reaction becomes unselective to the total hydrogenation to alkanes as follows:

$$
\begin{aligned}
& P d C_{x} H_{y}+2 C_{n} H_{2 n(\text { Chemisorb. })}+H_{2} \underset{D, E}{\rightarrow} P d C_{x} H_{y} \\
& +C_{n} H_{2 n(\text { gas })}+C_{n} H_{2 n+2(\text { gas })}
\end{aligned}
$$

Note that this reaction is not favored when $\mathrm{PdC}_{\mathrm{x}}$ species are present because the carbonaceous species avoid the participation of subsurface hydrogen in the reaction. On the other hand, the unselective formation of alkanes $(\mathrm{D}, \mathrm{E})$ can be conducted when the concentration of chemisorbed hydrogen is high yielding the formation of surface $\mathrm{PdH}_{\mathrm{x}}$. Therefore, $\beta-\mathrm{PdH}_{\mathrm{x}}$ is formed facilitating the production of $\mathrm{C}_{n} \mathrm{H}_{2 n}$ and $\mathrm{C}_{n} \mathrm{H}_{2 n+2}$ due to the participation of more reactive subsurface hydrogen in the hydrogenation of alkynes.

Open Access This article is distributed under the terms of the Creative Commons Attribution 4.0 International License (http://creativecommons.org/licenses/by/4.0/), which permits unrestricted use, distribution, and reproduction in any medium, provided you give appropriate credit to the original author(s) and the source, provide a link to the Creative Commons license, and indicate if changes were made. 